\title{
Hemocompatibility Evaluation of Biomaterials-The Crucial Impact of Analyzed Area
}

\author{
Johanna C. Clauser,* Judith Maas, Jutta Arens, Thomas Schmitz-Rode, Ulrich Steinseifer, \\ and Benjamin Berkels
}

Cite This: https://dx.doi.org/10.1021/acsbiomaterials.0c01589

Read Online

ACCESS | Lill Metrics \& More | 国 Article Recommendations | S1 Supporting Information

ABSTRACT: The hemocompatibility of blood-contacting medical devices remains one of the major challenges in medical device development. A common tool for the analysis of adherent and activated platelets on materials following in vitro tests is microscopy. Currently, most researchers develop their own routines, resulting in numerous different methods that are applied. The majority of those (semi-)manual methods analyze only a very small fraction of the material surface $(<1 \%)$, which neglects the inhomogeneity of platelet distribution and makes results hardly

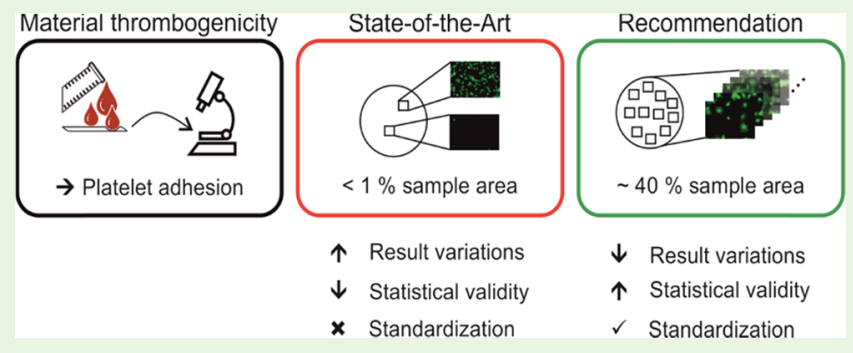
comparable. Within this study, we examined the relation between the fraction of analyzed sample area and the platelet adhesion result. By means of image segmentation and machine learning algorithms, 103100 microscopy images were analyzed automatically. We discovered a crucial impact of the analyzed surface fraction and thus a misrepresentation of a surface's platelet adhesion unless up to $40 \%$ of the sample surface is analyzed. These findings underline the necessity of standardization in the field of in vitro hemocompatibility tests and analyses in particular and provide a first basis to make future tests more reliable and comparable.

KEYWORDS: in vitro testing, standardization, fluorescence microscopy, platelet analysis, automation

\section{INTRODUCTION}

A growing percentage of elderly people in combination with a rising life expectancy increases the need for better and advanced medical care. Especially in the field of cardiovascular diseases, which include myocardial infarction, cardiac insufficiency, heart valve failure, and atherosclerotic alterations of the vascular system causing stenosis and occlusion of vessels, improvements are urgently needed since they caused $31 \%$ of deaths worldwide in 2016. ${ }^{1}$ Typical treatments of such diseases involve artificial heart valves, ventricular assist devices, grafts, or stents. ${ }^{2}$ These devices have in common that they are bloodcontacting medical devices. The biocompatibility and particularly the hemocompatibility of such devices is still the major challenge and the main restriction in their development. ${ }^{3}$ Hence, research and development in that area aim at improving the blood-material interaction by means of surface structuring, ${ }^{4,5}$ antithrombotic coatings, ${ }^{6}$ drug-eluting materials, ${ }^{7,8}$ and others. ${ }^{9,10}$ Besides the challenge from the material point of view, another difficulty is the lack of adequate in vitro investigation and analysis techniques. Although the standard DIN EN ISO 10993-4 provides several testing and analysis methods, these are more recommendations rather than clear instructions or regulations. ${ }^{11}$ Consequently, a wide range of test setups, ${ }^{12}$ test procedures, $^{6}$ and analysis techniques ${ }^{11}$ are applied for hemocompatibility evaluations, which makes results hardly comparable. The topic of standardizing test procedures was already addressed by several publications and studies in the past years; ${ }^{13}$ however, the issue with multiple analysis methods was not considered in detail so far.

Microscopy is a common method for the material analysis after in vitro hemocompatibility testing, as it allows for the distinct evaluation of the platelets' activation grade and their location of adherence. ${ }^{14-16}$ Nonetheless, optical platelet evaluation is broadly diversified with regard to microscopy techniques, ${ }^{17,18}$ platelet staining, ${ }^{19}$ positions of acquired images, ${ }^{20,21}$ appropriate fractions of analyzed sample area, $^{22,23}$ platelet counting, ${ }^{24}$ and platelet characterizing tools. $^{25}$ Again, the standard does not provide explicit instructions but only offers the possibility to choose any of the options. In consequence, researchers develop their own and individual protocols, which are hardly comparable with each other. Moreover, many groups fail to publish details about the applied analysis method, which finally makes comparisons impossible. ${ }^{14,26,27}$

Received: November 9, 2020

Accepted: January 13, 2021 
In this regard, the fraction of analyzed sample area and the classification of imaged platelets is probably the most important aspect that needs to be addressed as it directly and severely affects the result and the rating of a material's hemocompatibility. Since a time-consuming manual or semimanual platelet count is common practice for microscopy image analysis, the reported fractions of analyzed sample area are usually less than $0.9 \% .^{13,28-31}$ Only a few groups reported considerably more analyzed sample area with $14 \%^{32}$ and $33 \%{ }^{33}$ respectively. Taking the enormous number of platelets possibly adhering on $1 \mathrm{~cm}^{2}$ of material sample into account, it is hard to imagine that, according to the reported values, 0.009 $\mathrm{cm}^{2}$ represent the whole spectrum of platelet adhesion on this sample properly. Anyhow, to this point, it is unclear how inhomogeneous platelet adhesion is and how much sample area needs to be analyzed to get statistically valid results.

Accordingly, we hypothesize that the fraction of analyzed sample area strongly influences the result of the plateletcovered surface area as a measure for the hemocompatibility of a material. To evaluate this relation, we have conducted a static in vitro hemocompatibility study with different materials and made a statistical analysis of the variance and the deviation of the results depending on the analyzed sample area fraction.

\section{MATERIALS AND METHODS}

First, an in vitro hemocompatibility test series was conducted to create a database for the later statistical analysis. In the second step, fluorescence microscopy was used for platelet imaging and a previously developed automated analysis method was applied for platelet counting. ${ }^{34}$ Finally, the statistical analysis of the relation between result and analyzed sample area was carried out.

In Vitro Hemocompatibility Study. A static in vitro hemocompatibility test series was conducted using five different polymer materials (see Table 1) commonly used in hemocompatibility studies. ${ }^{35-38}$

Table 1. Materials for Hemocompatibility Testing

\begin{tabular}{|c|c|c|}
\hline ID & material & manufacturer \\
\hline PDMS & poly(dimethyl siloxane) & $\begin{array}{l}\text { Bess Medizintechnik GmbH } \\
\text { Germany }\end{array}$ \\
\hline PTFE & poly(tetrafluoro ethylene) & $\begin{array}{l}\text { Bess Medizintechnik } \mathrm{GmbH} \text {, } \\
\text { Germany }\end{array}$ \\
\hline PET & poly(ethylene terephthalate) & Thermo Fisher Scientific \\
\hline $\mathrm{PCU}_{\text {extr }}$ & $\begin{array}{l}\text { poly (carbonate urethane)- } \\
\text { extrusion }\end{array}$ & Lubrizol (raw material) \\
\hline $\mathrm{PCU}_{\text {cast }}$ & $\begin{array}{l}\text { poly (carbonate urethane)- } \\
\text { mold casting }\end{array}$ & Lubrizol (raw material) \\
\hline
\end{tabular}

Poly(dimethyl siloxane) (PDMS), poly(tetrafluoro ethylene) (PTFE), and poly(ethylene terephthalate) (PET) were purchased ready-to-use, whereas poly(carbonate urethane) (PCU) extr and $\mathrm{PCU}_{\text {cast }}$ were manufactured out of the purchased polymer granulate. Extrusion processing of $\mathrm{PCU}_{\text {extr }}$ was done at the Institute of Plastics Processing, RWTH Aachen University, as described elsewhere. ${ }^{35,39}$ For mold casting of $\mathrm{PCU}_{\text {cast }}$ the $\mathrm{PCU}$ granulate was dissolved in dimethylacetamide (DMAC) in a $1: 9 \mathrm{wt} \%$ ratio for $24 \mathrm{~h}$ with gentle rotation. Ahead of casting, any air bubbles were removed from the PCU solution by centrifugation of small portions for $10 \mathrm{~min}$ at 4000 $\times g$ relative centrifugal force. PCU solution $(6 \mathrm{~g})$ was weighed into a horizontally adjusted mold with a plain silicon wafer (Micromotive $\mathrm{GmbH}, \mathrm{Germany})$. The mold was placed in a vacuum oven (Hereaus VT $6060 \mathrm{M}$, VWR, Germany), and temperature and pressure were adjusted as shown in Table 2. During that process, the DMAC evaporates and the PCU foil remains. After carefully removing from the mold, the foils rested for at least 1 week to evaporate from remaining DMAC. ${ }^{38}$
Table 2. Mold Casting Parameter ${ }^{38}$

$\begin{array}{cccc}\text { step } & \text { time }(\mathrm{min}) & \text { temperature }\left({ }^{\circ} \mathrm{C}\right) & \text { pressure }(\mathrm{mbar}) \\ 1 & 30 & 20 & 1013 \\ 2 & 60 & 20 & 700 \\ 3 & 480 & 60 & 700\end{array}$

For each of the $n=10$ test repetitions, duplicates of ethylene-oxidesterilized materials were used. For the tests, fresh blood was drawn from healthy donors, who were free of any platelet-affecting drugs, from the cubital vein without stowing (Ethical Committee reference number EK 348/16 and EK 033/18). The blood was immediately anticoagulated with $3.2 \%$ citrate (S-Monovette, Sarstedt, Germany) and further processed to platelet-rich plasma (PRP) with a thrombocrit of $50000 \mathrm{PLT} / \mu \mathrm{L}$. Round material samples with a diameter of $11 \mathrm{~mm}$ were incubated with PRP for $1 \mathrm{~h}$ at $37{ }^{\circ} \mathrm{C}$ statically in well plates. Afterward, PRP was carefully removed, material samples were washed multiple times with phosphate-buffered saline (Lonza, Germany) and incubated with $2 \%$ glutaraldehyde (Roth, Germany) for unspecific platelet staining. The material samples were mounted on microscopy slides with coverslips on top by means of mowiol (Roth, Germany), stored in the dark at room temperature for $12 \mathrm{~h}$ and afterward at $8{ }^{\circ} \mathrm{C}$ for a minimum of 3 days prior to microscopy. A more detailed test procedure is described elsewhere. $^{13,38}$

Microscopy and Image Analysis. Fluorescence microscopy of the material samples was performed using an Observer $\mathrm{Z} 1$ microscope (Carl Zeiss GmbH, Germany) with a 40-fold magnification (resolution/pixel: $0.114 \times 0.114 \mu \mathrm{m}^{2}$ ). To maximize the imaged sample area, tile images with $5 \times 5$ single images per tile were recorded. With a single image area of $78969 \mu \mathrm{m}^{2}$, the total tile area results to $1.95 \times 106 \mu \mathrm{m}^{2}$. Tiles were placed randomly on the samples only neglecting areas of obvious contamination by, e.g., glutaraldehyde residues or mowiol, resulting in a maximum of 45 tiles per sample, representing $92.34 \%$ sample surface (cf. Figure 1).

Prior to image acquisition, nine focus points/tile were adjusted manually to reduce out-of-focus images within the tiles due to slight height differences of the mounted material samples. Except PET (M3), all materials were imaged with $100 \%$ fluorescence light intensity, whereas intensity was reduced to $58.32 \%$ for PET because of strong autofluorescence in the recorded green spectrum $(509 \mathrm{~nm}$ emission wavelength).

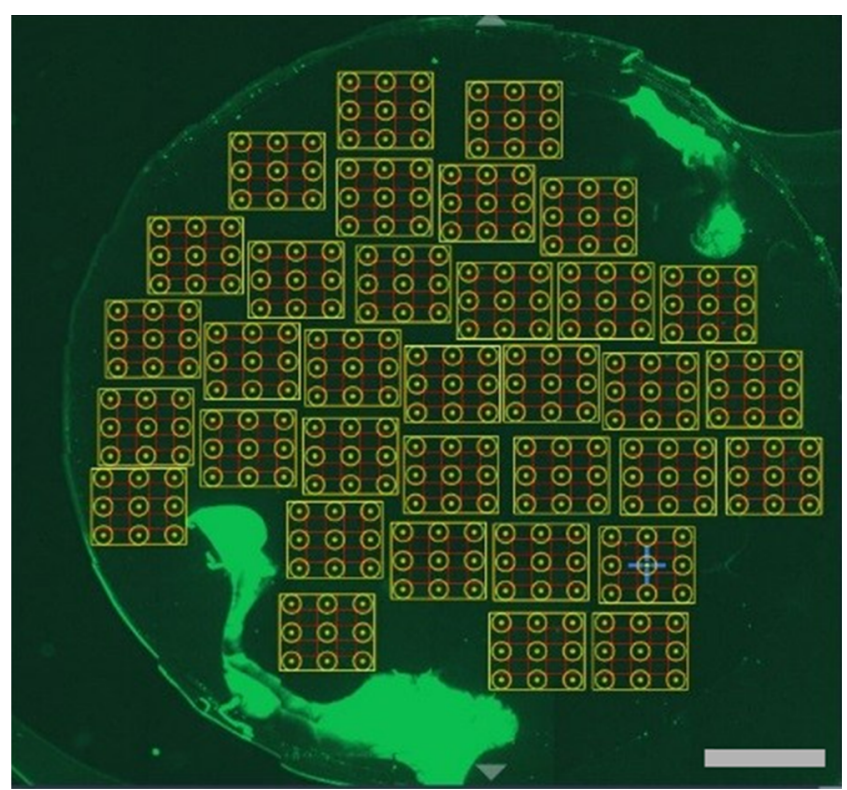

Figure 1. Tile placement with focus points on material sample; scale bar $\triangleq 2000 \mu \mathrm{m}$. 


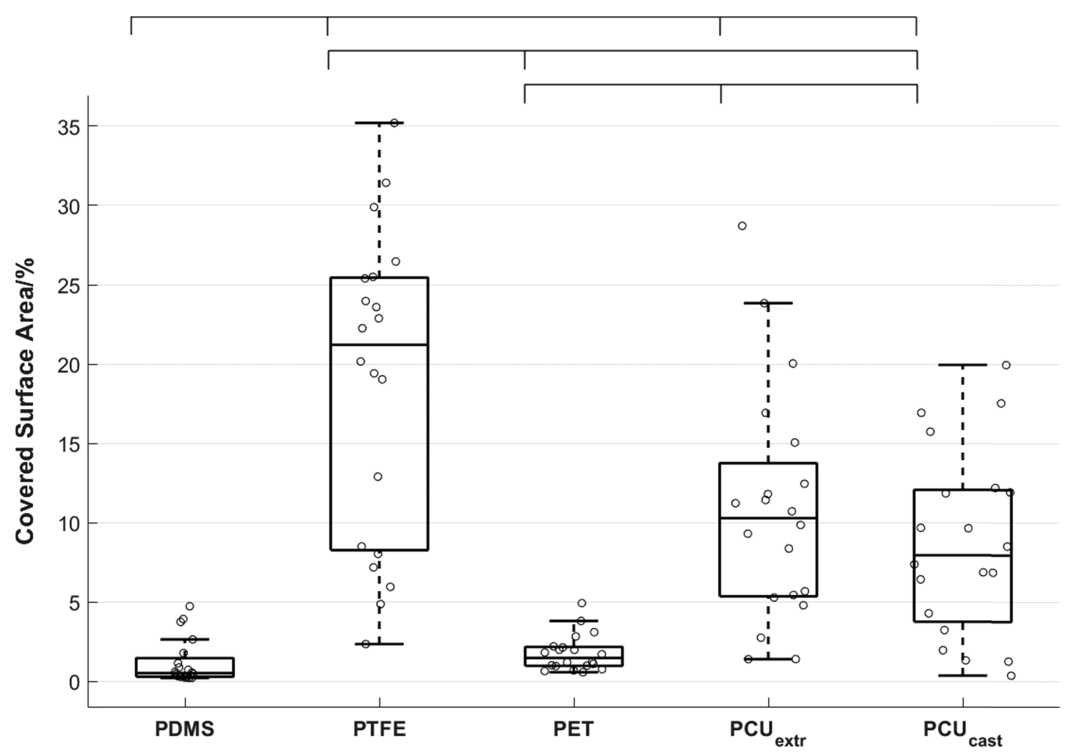

Figure 2. Platelet-covered surface area; boxplots including all individual data points, crossbars for $p<0.05 .^{38}$

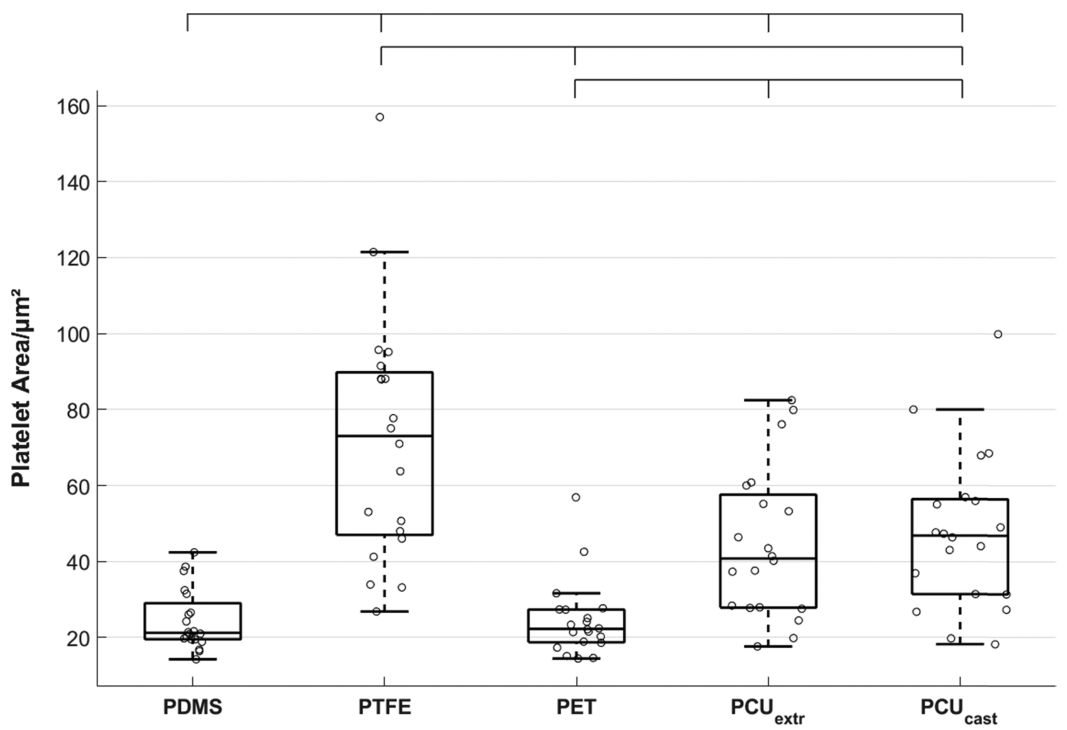

Figure 3. Area per platelet; boxplots including all individual data points, crossbars for $p<0.05^{38}$

All microscopy images were analyzed by means of a newly developed automated image analysis method. ${ }^{34,38}$ In short, image quality was checked in a manual incoming inspection excluding images that were out of focus, overexposed, or heavily contaminated. Afterward, an image segmentation algorithm was applied to identify and parameterize all single components on every image. Connected platelet aggregates were treated as one component. In the following, a random forest machine learning algorithm categorized each component into the class "platelet" or "no platelet." Those components categorized as "platelets" were then analyzed with regard to number and size and the covered surface area, area per platelet, and platelet density were calculated. In total, 103100 images were included for platelet evaluation and statistical analysis.

Statistical Analysis. A measure of inhomogeneity was calculated to prove the hypothesis that platelet distribution is inhomogeneous across a material sample. For each sample, the difference of the platelet-covered surface area CovDiff of one single image $A_{\text {covered }}(i)$ to the total covered area $A_{\text {covered }}$ (total), both as percentage related to the corresponding area, was calculated (eq 1).

$$
\operatorname{CovDiff}(i)=\mid A_{\text {covered }}(i)-A_{\text {covered }}(\text { total }) \mid
$$

To ensure comparability, the value of CovDiff was then related to the total covered area of the corresponding sample and expressed as percentage (eq 2). In the case of high CovDiff values and low total covered area values, the inhomogeneity may exceed values of $100 \%$ considerably.

$$
\text { inhomogeneity }=\frac{\operatorname{CovDiff}(i)}{A_{\text {covered }}(\text { total })}
$$

The impact of analyzed sample area on the result of covered surface area was determined by randomly choosing subsets of $k$ images representing different fractions of analyzed surface area and evaluating the covered surface area on these images. The procedure was carried out for analyzed surface fractions from $0.1 \%$ ( 1 microscope image) to 60-90\%, depending on the existing amount of images for each material sample. To highlight the impact of inhomogeneous platelet distribution, the choice of $k$ images was repeated 10 times for every surface fraction, whereby all images were included in each round. The coefficient of variance $(\mathrm{CV})$ was calculated for each surface fraction. Additionally, mean values and standard deviations were normalized to the final "true" value, given by the analysis of all images from each 


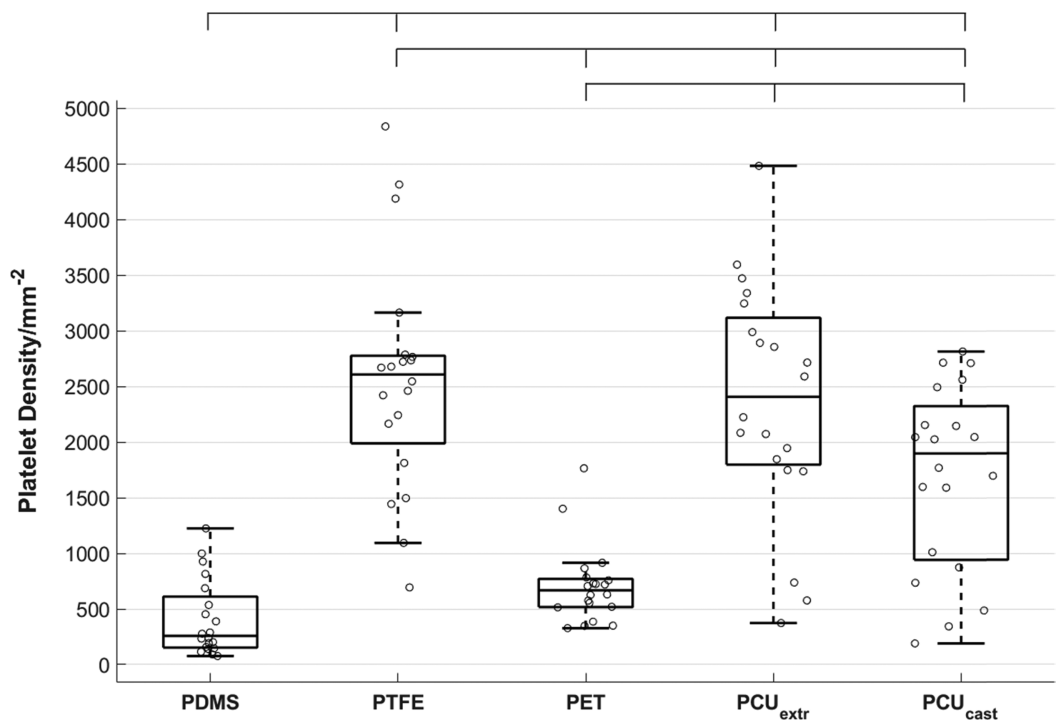

Figure 4. Platelet density; boxplots including all individual data points, crossbars for $p<0.05 .^{38}$

data group. Calculations were performed using Matlab R2017b (MathWorks).

\section{RESULTS}

First, the overall results of the hemocompatibility test series will be presented briefly, followed by the inhomogeneity evaluation and the impact of analyzed sample area on the results. Finally, a threshold of necessarily analyzed sample area is calculated.

Hemocompatibility Study. The results of the hemocompatibility study are presented in boxplots with crossbars indicating significant differences with $p<0.05$ according to one-way analysis of variance (ANOVA) and Tukey-HSD post hoc test for normally distributed data and Kruskal-Wallis test for non-normally distributed data based on the Shapiro-Wilk test (all IBM SPSS Statistics 24, IBM). The results are based on all acquired images that passed the incoming control.

Figure 2 shows the covered surface area of the five different materials. PTFE reveals the most platelet adhesion with 18.75 \pm 9.50\%, whereas PDMS and PET show the least platelet adhesion below 2\%. Both PCU materials are in the middle range with about $10 \%$ area covered with platelets. Except for

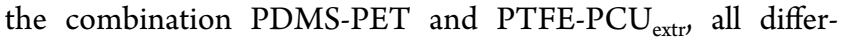
ences among the materials are rated statistically significant $(p<$ $0.05)$.

A similar result is obvious for the area per platelet as shown in Figure 3. The relation between the different materials is the same as for the covered area with PTFE $\left(72.25 \pm 31.66 \mu \mathrm{m}^{2}\right)$ at the top, PDMS and PET at the bottom of the scale $(\sim 25$ $\left.\mu \mathrm{m}^{2}\right)$, and the PCUs $\left(\sim 45 \mu \mathrm{m}^{2}\right)$ in between. This is consistent since a larger area per platelet indicates a stronger platelet activation, which goes along with more covered surface area.

The platelet density (Figure 4) shows a slightly different behavior with the mean value of $\mathrm{PCU}_{\text {extr }}$ being more close to PTFE with $2376 \pm 1031 \mathrm{~mm}^{2}$ and $2563 \pm 1008 \mathrm{~mm}^{2}$, respectively. However, differences are still statistically significant except PDMS-PET, which reveal again the least platelet density below $1000 \mathrm{~mm}^{2}$. Results are in line with the previous numbers, since a higher platelet density represents a larger number of adherent platelets and thus a higher thrombogenic potential of a material.
Inhomogeneity Evaluation. The maximum inhomogeneity for each material group is shown in Table 3 .

Table 3. Inhomogeneity Per Material

\begin{tabular}{lccccc} 
& PDMS & PTFE & PET & PCU $_{\text {extr }}$ & PCU $_{\text {cast }}$ \\
inhomogeneity $_{\max }(\%)$ & 2023 & 799 & 1068 & 1653 & 1365 \\
\hline
\end{tabular}

Inhomogeneity is extremely high for all materials with values up to $2000 \%$, which means that the platelet-covered surface area was 20 times higher or lower on one image compared to the overall final value of covered surface area.

As a visual example, Figure 5 shows two exemplary images located diagonally to each other on the same material sample $\left(\mathrm{PCU}_{\text {cast }}\right)$. With $0.35 \%$ and $32.79 \%$ covered surface area, respectively, the platelet distribution is immensely inhomogeneous. Additionally, the overall covered surface area on this sample lies with $9.67 \%$ far away from both images.

Analyzed Sample Area Fraction. The influence of inhomogeneous platelet distribution on the actual covered surface area as a function of analyzed sample area was evaluated for each material and each test day individually (data not shown). As an example, Figure 6 shows the coefficient of variance $(\mathrm{CV})$ from $i=10$ random choices as a function of the analyzed samples area for $\mathrm{PCU}_{\text {cast }}$ (test day 8 ).

A two-term power fit describes the behavior of the CV. It is striking that the $\mathrm{CV}$ reaches values of $>0.5$ and higher for analyzed sample fractions below 2\%. For rising sample fractions, it drops and nearly reaches a plateau value in the region of 0.1 . Figure 7 depicts the compiled $\mathrm{CVs}$ for $\mathrm{PCU}_{\text {cast }}$ from every blood test repetition. The compilations for the other materials can be found in the Supporting Information (Figures S1-S4).

Again, small analyzed area fractions correlate with high CVs, whereas CVs decrease with increasing analyzed surface area. Interestingly, the deviation from the two-term power fit, which describes the course of the $\mathrm{CV}$, is reduced after $20 \%$ sample area is analyzed for $\mathrm{PCU}_{\text {cast }}$ as well as the other materials (see the Supporting Information). Of note, PTFE (Figure S2) and PET (Figure S3) show generally less variation compared to the other three materials. 

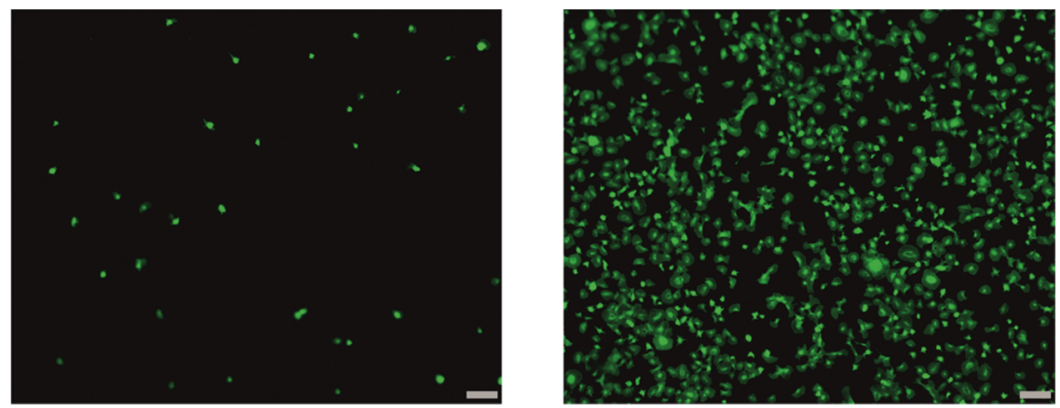

Figure 5. Exemplary microscope images from the same sample $\left(\mathrm{PCU}_{\text {cast }}\right)$ with $0.35 \%$ covered surface area (left) and $32.79 \%$ covered surface area (right); scale bar $\triangleq 20 \mu \mathrm{m}$.

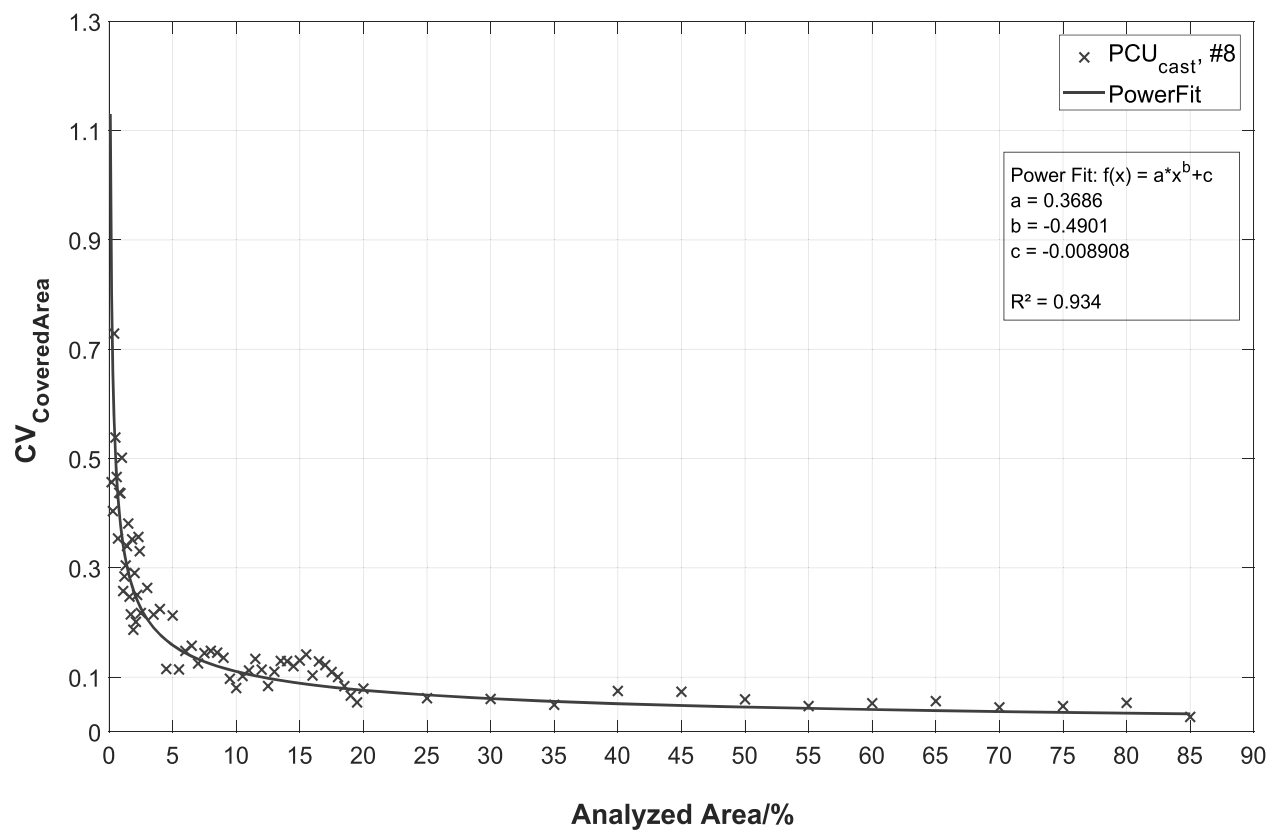

Figure 6. $\mathrm{CV}$ as a function of analyzed sample area plus two-term power fit for $\mathrm{PCU}_{\text {cast }} \# 8$.

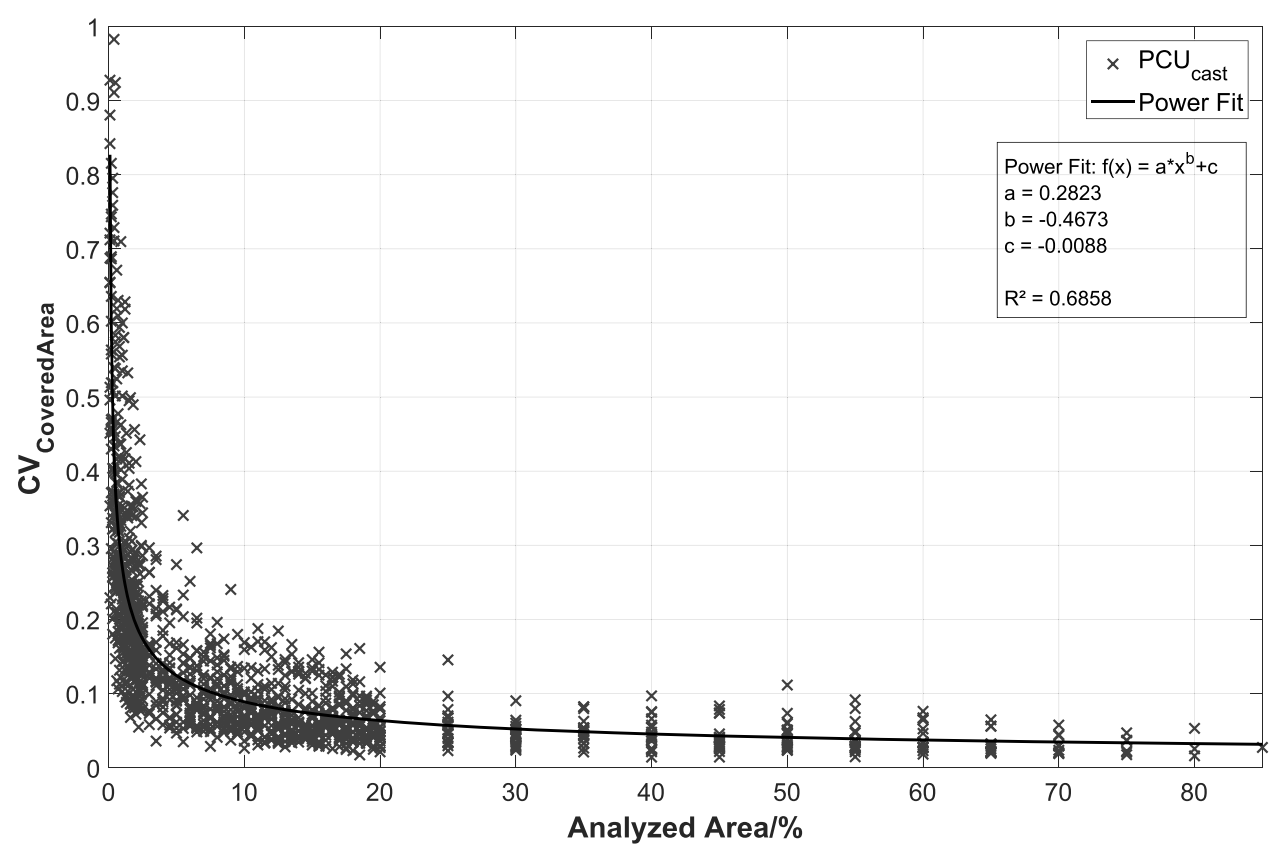

Figure 7. Combined CV data for $\mathrm{PCU}_{\text {cast }}$. 


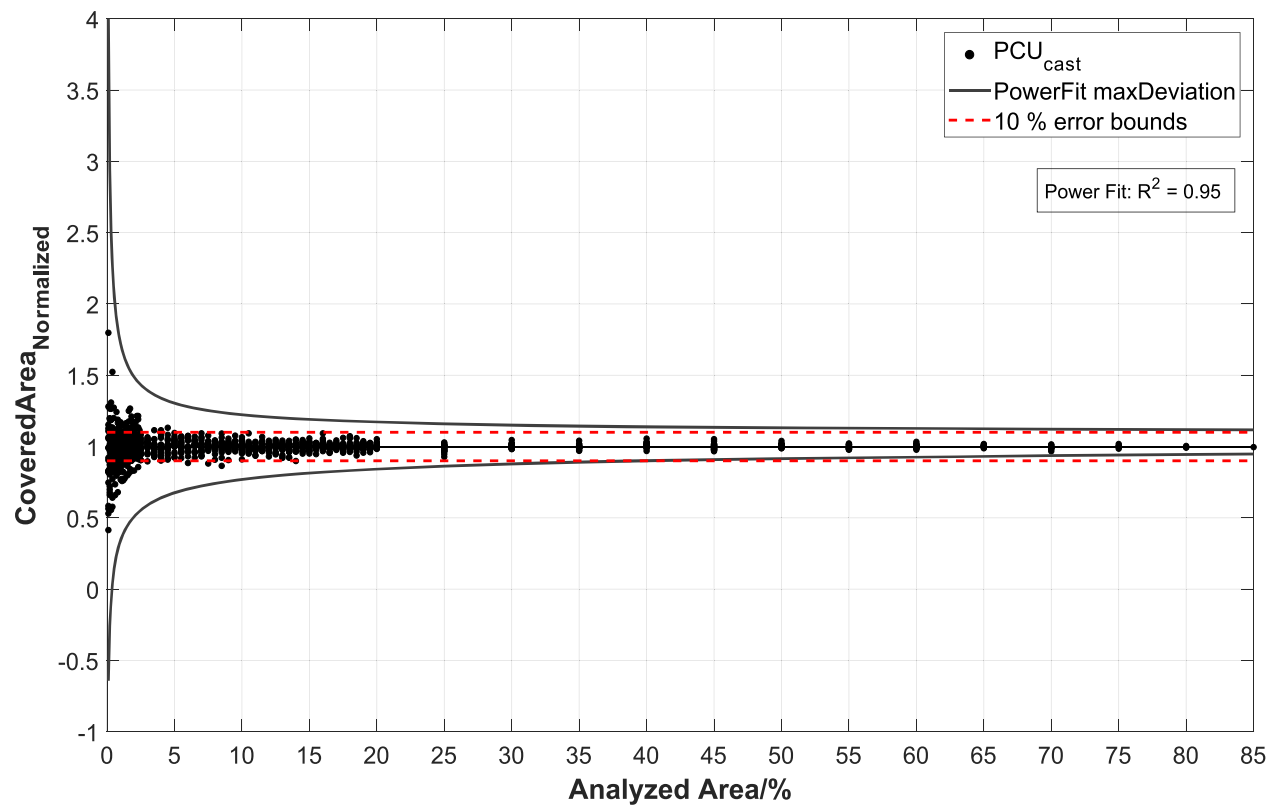

Figure 8. Covered surface area of $\mathrm{PCU}_{\text {cast }}$ as a function of the analyzed sample area, normalized to the overall value; curve fits for the maximum deviations and dotted lines for $10 \%$ error bounds.

Area Threshold. The threshold of necessarily analyzed sample area fraction was determined by taking the deviations from the 10 times repeated random analysis to the overall result into account. Figure 8 shows the data for $\mathrm{PCU}_{\text {cast }}$ the power fit describing the course of the maximum deviations and the $\pm 10 \%$ error bounds. Corresponding to the CV data, the deviations are higher for small analyzed sample fractions and decrease to a plateau value with increasing analyzed area. The other materials reveal a comparable behavior, which only differs with regard to the $x$-value where error bounds and deviation curve fits intersect. The corresponding figures are presented in the Supporting Information (Figures S5-S8).

Since $\pm 10 \%$ error and deviation are regarded as acceptable for variations of biologic material like blood and measurement errors of devices such as blood count analyzer are in a similar range, ${ }^{40,41}$ this error bound was chosen for the area threshold. In particular, the lower error bound of $-10 \%$ was considered as this represents the underestimation of covered surface area and hence platelet adhesion. The intersection values of deviation curve fits and the 0.9 error bound are shown in Table 4 for each material.

Table 4. Area Threshold Related to 0.9 Error Bound

\begin{tabular}{|cccccc|}
\hline & PDMS & PTFE & PET & PCU $_{\text {extr }}$ & PCU $_{\text {cast }}$ \\
\hline area threshold (\%) & 34 & 30 & 14 & 36 & 40 \\
\hline
\end{tabular}

The majority of materials reveals an area threshold between $30 \%$ (PTFE) and 40\% ( PCU $_{\text {cast }}$ ) of analyzed sample area; however, the threshold for PET is severely lower with $14 \%$.

\section{DISCUSSION}

The results of the hemocompatibility study reflect the expected results as PTFE presents the highest degree of platelet adhesion and activation, whereas PDMS and PET show the least tendency of platelet adhesion. Notably, the standard deviation within each material group is quite high although the results are based on duplicates of $n=10$ test repetitions and the use of a standard test protocol. ${ }^{13}$ This highlights the importance of standard test and analysis protocols, which reduce additional error sources. Since the aim of the study was not the distinct analysis of the different materials but more the general analysis modalities, the material-specific results serve only as a control that the in vitro tests are consistent with the literature. $^{13,42-46}$

The inhomogeneity evaluation confirms the optical impression that platelets are spread randomly all over the different material samples. Consequently, there are regions and microscopy images with a large amount of adherent platelets or with nearly no adherent platelets. The calculated maximum inhomogeneity even exceeds $2000 \%$ compared to the overall result, which emphasizes the necessity to analyze a wide area of sample surface. By imaging only a very small fraction of the evaluated material sample, the chance for a biased result in either direction is very high. This is illustrated by the CV as a function of analyzed sample area. CVs of $0.5-1$ for area fractions below $2 \%$ do not depict statistically credible results. Interestingly, the $\mathrm{CV}$ can be described in a two-term power function as it drops fast within the first 5\% analyzed sample area. In the following, the decrease slows down until nearly a plateau is reached. This means that there is a certain fraction of analyzed sample area, which serves as a threshold after that the $\mathrm{CV}$ cannot be reduced anymore. Hence, the remaining variations are due to, e.g., variations in the blood or the laboratory equipment.

To determine this area threshold, the normalized standard deviations were plotted as a function of analyzed sample fraction. Again, a two-term power fit describes the course of the deviations well. The intersection of the deviation curve fit and $10 \%$ error bounds from the actual result (result based on the analysis of every microscope image) depict the necessarily analyzed sample area fraction and thus the area threshold. More precisely, the lower error bound was considered for this purpose. In the context of hemocompatibility evaluation, the underestimation of the platelet adhesion tendency on a material is considerably more severe than a slight over- 
estimation. The latter includes an extra safety, whereas a falsepositive rating could have serious consequences on the performance of medical devices and patient safety.

Interestingly, the five different materials present different area thresholds. The deviation and thus the inhomogeneity on $\mathrm{PCU}_{\text {cast }}$ seem to be highest as the area threshold is $40 \%$. PTFE reveals a threshold of $30 \%$ analyzed sample area, which is lower but still in a comparable range, while the thresholds for PDMS and $\mathrm{PCU}_{\text {extr }}$ are located in between. With $14 \%$ area threshold, PET is considerably lower than all other materials, which implies that platelet distribution is less inhomogeneous on PET since less sample area needs to be analyzed to fall below the limit of $10 \%$ error. An explanation for this phenomenon is only speculative but could be due to the material properties of the polymers. Although microscopy of the materials prior to hemocompatibility testing did not reveal any surface defects, minor material differences could result from the fabrication or the polymerization of the materials.

However, the area threshold results underline the need for regulations of biomaterial analysis. Analyzing marginal sample area fractions does not provide reliable, reproducible, and statistically valid results. Based on the presented results of this study, our current recommendation is to analyze $40 \%$ of the sample area, as this seems to be a maximum of necessarily analyzed sample area, at least for the materials investigated in this study.

The presented study reveals important details for the design of future hemocompatibility test and provides a first step toward the standardization of optical platelet adhesion assessment. Nonetheless, the transferability and general applicability have to be evaluated in the future. Within this study, five different polymers were evaluated. To figure out if area thresholds differ between these materials and other polymers or even other material groups like metals, further materials have to be tested and evaluated. Additionally, the thrombocrit of $50000 \mathrm{PLT} / \mu \mathrm{L}$ as well as the sample size might influence the inhomogeneous platelet distribution. It has to be verified if the platelet distribution is more or less inhomogeneous on larger and smaller material samples and if different sample sizes require different analysis area thresholds.

Furthermore, the method that was used to automatically analyze the microscopy images is tailored to specific parameters. $^{38}$ Identical platelet staining, image resolution, and magnification are crucial to apply the automated analysis method to any microscope image. Hence, consistency of these parameters has to be maintained. The transferability of the method as an important tool for the standardized material evaluation is part of future work.

\section{CONCLUSIONS}

An extensive statistical analysis was carried out focusing on the relationship between analyzed sample area fraction and the resulting hemocompatibility results after static in vitro hemocompatibility testing. A large database of more than 100000 microscopy images was set up from 10 test repetitions with duplicates of five different material samples. It was shown that platelet distribution is immensely inhomogeneous on the material samples with up to $2000 \%$ deviation from the actual result. Based on this finding, the area threshold for a result deviation within $10 \%$ error bounds was determined for each material. Thresholds vary between $14 \%$ and $40 \%$, which is both far above the analyzed area fractions described in other publications. These results put emphasis on the need for clear regulations and standards for in vitro hemocompatibility testing and analyses. Without such rules, results will always lack comparability, reproducibility, and partly even reliability. In the future, the determined area thresholds should be taken into consideration when optical platelet adhesion evaluations are performed.

\section{ASSOCIATED CONTENT}

\section{(s) Supporting Information}

The Supporting Information is available free of charge at https://pubs.acs.org/doi/10.1021/acsbiomaterials.0c01589.

Combined CV data for PDMS (Figure S1); combined $\mathrm{CV}$ data for PTFE (Figure S2); combined CV data for PET (Figure S3); combined CV data for $\mathrm{PCU}_{\text {extr }}$ (Figure S4); covered surface area of PDMS as a function of the analyzed sample area, normalized to the overall value; curve fits for the maximum deviations and dotted lines for $10 \%$ error bounds (Figure S5); covered surface area of PTFE as a function of the analyzed sample area, normalized to the overall value; curve fits for the maximum deviations and dotted lines for $10 \%$ error bounds (Figure S6); covered surface area of PET as a function of the analyzed sample area, normalized to the overall value; curve fits for the maximum deviations and dotted lines for 10\% error bounds (Figure S7); and covered surface area of $\mathrm{PCU}_{\text {extr }}$ as a function of the analyzed sample area, normalized to the overall value; curve fits for the maximum deviations and dotted lines for $10 \%$ error bounds (Figure S8)

(PDF)

\section{AUTHOR INFORMATION}

\section{Corresponding Author}

Johanna C. Clauser - Department of Cardiovascular Engineering, Institute of Applied Medical Engineering, Medical Faculty, RWTH Aachen University, 52074 Aachen, Germany; 10 orcid.org/0000-0002-9050-2391; Phone: +49 241 8089356; Email: clauser@ame.rwth-aachen.de; Fax: +492418082442

\section{Authors}

Judith Maas - Department of Cardiovascular Engineering, Institute of Applied Medical Engineering, Medical Faculty, RWTH Aachen University, 52074 Aachen, Germany

Jutta Arens - Department of Cardiovascular Engineering, Institute of Applied Medical Engineering, Medical Faculty, RWTH Aachen University, 52074 Aachen, Germany; Chair in Engineering Organ Support Technologies, Department of Biomechanical Engineering, Faculty of Engineering Technology, University of Twente, 7522 NB Enschede, The Netherlands

Thomas Schmitz-Rode - Department of Cardiovascular Engineering, Institute of Applied Medical Engineering, Medical Faculty, RWTH Aachen University, 52074 Aachen, Germany

Ulrich Steinseifer - Department of Cardiovascular Engineering, Institute of Applied Medical Engineering, Medical Faculty, RWTH Aachen University, 52074 Aachen, Germany

Benjamin Berkels - AICES Graduate School and Institute for Geometry and Practical Mathematics, RWTH Aachen University, 52062 Aachen, Germany 
Complete contact information is available at:

https://pubs.acs.org/10.1021/acsbiomaterials.0c01589

\section{Funding}

Parts of this project were funded by the INTERREG Program V-A Euregio Maas-Rhine of the European Union (grant no. 2016/98602). B.B. was funded in part by the Excellence Initiative of the German Federal and State Governments through grant GSC 111.

\section{Notes}

The authors declare no competing financial interest.

\section{ACKNOWLEDGMENTS}

The authors thank Dr. Doris Keller from the University Medical Center RWTH Aachen University for the blood withdrawal; Florian Petzinka from the Institute of Plastics Processing RWTH Aachen University for the extrusion of PCU foils; and the Institute for Medical Statistics of the University Hospital RWTH Aachen for the advice during the statistical data analysis. Image analyses were performed with computing resources granted by RWTH Aachen University under project rwth0314. This article comprises results and figures that are part of the $\mathrm{PhD}$ Thesis from J.C.C., submitted in 2019 at RWTH Aachen University, Germany. ${ }^{38}$

\section{REFERENCES}

(1) World Health Organization. Cardiovascular Diseases (CVDs). https://www.who.int/en/news-room/fact-sheets/detail/ cardiovascular-diseases-(cvds).

(2) Jaffer, I. H.; Fredenburgh, J. C.; Hirsh, J.; Weitz, J. I. Medical device-induced thrombosis: what causes it and how can we prevent it? J. Thromb. Haemostasis 2015, 13, S72-S81.

(3) Brash, J. L.; Horbett, T. A.; Latour, R. A.; Tengvall, P. The blood compatibility challenge. Part 2: Protein adsorption phenomena governing blood reactivity. Acta Biomater. 2019, 94, 11-24.

(4) Pham, T. T.; Wiedemeier, S.; Maenz, S.; Gastrock, G.; Settmacher, U.; Jandt, K. D.; Zanow, J.; Lüdecke, C.; Bossert, J. Hemodynamic aspects of reduced platelet adhesion on bioinspired microstructured surfaces. Colloids Surf., B 2016, 145, 502-509.

(5) Liu, R.; Qin, Y.; Wang, H.; Zhao, Y.; Hu, Z.; Wang, S. The in vivo blood compatibility of bio-inspired small diameter vascular graft: effect of submicron longitudinally aligned topography. BMC Cardiovasc. Disord. 2013, 13, No. 79.

(6) Liu, C.; Lin, Z.; Qiao, C.; Zhao, Z.; Wang, C.; Sun, X.; Shi, Y. Hemocompatibility assay of a micro-catheter using hydrophilic coating biomaterials. Bio-Med. Mater. Eng. 2019, 30, 1-9.

(7) Huang, L.-Y.; Yang, M.-C.; Tsou, H.-M.; Liu, T.-Y. Hemocompatibility and anti-fouling behavior of multilayer biopolymers immobilized on gold-thiolized drug-eluting cardiovascular stents. Colloids Surf., B 2019, 173, 470-477.

(8) Shitole, A. A.; Giram, P. S.; Raut, P. W.; Rade, P. P.; Khandwekar, A. P.; Sharma, N.; Garnaik, B. Clopidogrel eluting electrospun polyurethane/polyethylene glycol thromboresistant, hemocompatible nanofibrous scaffolds. J. Biomater. Appl. 2019, 33, $1327-1347$.

(9) Feng, Y.; Zhao, H.; Behl, M.; Lendlein, A.; Guo, J.; Yang, D. Grafting of poly(ethylene glycol) monoacrylates on polycarbonateurethane by UV initiated polymerization for improving hemocompatibility. J. Mater. Sci.: Mater. Med. 2013, 24, 61-70.

(10) Sask, K. N.; Berry, L. R.; Chan, A. K. C.; Brash, J. L. Modification of Polyurethane Surface with an Antithrombin-Heparin Complex for Blood Contact: Influence of Molecular Weight of Polyethylene Oxide Used as a Linker/Spacer. Langmuir 2012, 28, 2099-2106.
(11) Biological Evaluation of Medical Devices - Part 4: Selection of Tests for Interactions with Blood; Deutsches Institut furr Normung e.V., Berlin, 2017, 11.100.20 (10993-4).

(12) Braune, S.; Latour, R. A.; Reinthaler, M.; Landmesser, U.; Lendlein, A.; Jung, F. In Vitro Thrombogenicity Testing of Biomaterials. Adv. Healthcare Mater. 2019, 8, No. 1900527.

(13) Braune, S.; Sperling, C.; Maitz, M. F.; Steinseifer, U.; Clauser, J.; Hiebl, B.; Krajewski, S.; Wendel, H. P.; Jung, F. Evaluation of platelet adhesion and activation on polymers: Round-robin study to assess inter-center variability. Colloids Surf., B 2017, 158, 416-422.

(14) Wang, J.; Gao, L.; Li, Y.; Liu, B. Experimental research on laser interference micro/nano fabrication of hydrophobic modification of stent surface. Lasers Med. Sci. 2017, 32, 221-227.

(15) Braune, S.; Gros, M.; Walter, M.; Zhou, S.; Dietze, S.; Rutschow, S.; Lendlein, A.; Tschoepe, C.; Jung, F. Adhesion and activation of platelets from subjects with coronary artery disease and apparently healthy individuals on biomaterials. J. Biomed. Mater. Res., Part B 2016, 104, 210-217.

(16) Weber, M.; Steinle, H.; Golombek, S.; Hann, L.; Schlensak, C.; Wendel, H. P.; Avci-Adali, M. Blood-Contacting Biomaterials: In Vitro Evaluation of the Hemocompatibility. Front. Bioeng. Biotechnol. 2018, 6, No. 99.

(17) Michikoshi, J.; Matsumoto, S.; Miyawaki, H.; Morita, M.; Niu, H.; Seo, K.; Hagiwara, S.; Kitano, T. Evaluation of Proteins and Cells that Adsorb to Dialysis Membranes Used in Continuous Hemodiafiltration: Comparison of AN69ST, Polymethylmethacrylate, and Polysulfone Membranes. Blood Purif. 2019, 48, 358-367.

(18) Yuan, W.; Feng, Y.; Wang, H.; Yang, D.; An, B.; Zhang, W.; Khan, M.; Guo, J. Hemocompatible surface of electrospun nanofibrous scaffolds by ATRP modification. Mater. Sci. Eng., C 2013, 33, 3644-3651.

(19) Sivaraman, B.; Latour, R. A. The relationship between platelet adhesion on surfaces and the structure versus the amount of adsorbed fibrinogen. Biomaterials 2010, 31, 832-839.

(20) Navitsky, M. A.; Taylor, J. O.; Smith, A. B.; Slattery, M. J.; DEUTSCH, S.; Siedlecki, C. A.; Manning, K. B. Platelet adhesion to polyurethane urea under pulsatile flow conditions. Artif. Organs 2014, $38,1046-1053$.

(21) Zhou, M.; Yang, J. H.; Ye, X.; Zheng, A. R.; Li, G.; Yang, P. F.; Zhu, Y.; Cai, L. Blood Platelet's Behavior on Nanostructured Superhydrophobic Surface. J. Nano Res. 2008, 2, 129-136.

(22) Klein, M.; Kuhn, Y.; Woelke, E.; Linde, T.; Ptock, C.; Kopp, A.; Bletek, T.; Schmitz-Rode, T.; Steinseifer, U.; Arens, J.; et al. In-vitro Study on the Hemocompatibility of Plasma Electrolytic Oxidation Coatings on Titanium Substrates. Artif. Organs 2020, 44, 419-427.

(23) Jia, R. P.; Zong, A. X.; He, X. Y.; Wei, L. Synthesis and Blood Compatibility of Fluorinated Polyurethane Elastomer. Appl. Mech. Mater. 2014, 685, 473-476.

(24) Ye, W.; Shi, Q.; Wong, S.-C.; Hou, J.; Xu, X.; Yin, J. Precise patterning of the SEBS surface by UV lithography to evaluate the platelet function through single platelet adhesion. Biomater. Sci. 2014, 2,1186 .

(25) Braune, S.; Alagöz, G.; Seifert, B.; Lendlein, A.; Jung, F. Automated image-based analysis of adherent thrombocytes on polymer surfaces. Clin. Hemorheol. Microcirc. 2012, 52, 349-355.

(26) Ding, Y.; Leng, Y.; Huang, N.; Yang, P.; Lu, X.; Ge, X.; Ren, F.; Wang, K.; Lei, L.; Guo, X. Effects of microtopographic patterns on platelet adhesion and activation on titanium oxide surfaces. J. Biomed. Mater. Res., Part A 2013, 101A, 622-632.

(27) He, C.; Wang, M.; Cai, X.; Huang, X.; Li, L.; Zhu, H.; Shen, J.; Yuan, J. Chemically induced graft copolymerization of 2-hydroxyethyl methacrylate onto polyurethane surface for improving blood compatibility. Appl. Surf. Sci. 2011, 258, 755-760.

(28) Milner, K. R.; Snyder, A. J.; Siedlecki, C. A. Sub-micron texturing for reducing platelet adhesion to polyurethane biomaterials. J. Biomed. Mater. Res., Part A 2006, 76A, 561-570.

(29) Zhao, J.; Song, L.; Yin, J.; Ming, W. Anti-bioadhesion on hierarchically structured, superhydrophobic surfaces. Chem. Commun. 2013, 49, 9191-9193. 
(30) Lutter, C.; Nothhaft, M.; Rzany, A.; Garlichs, C. D.; Cicha, I. Effect of specific surface microstructures on substrate endothelialisation and thrombogenicity: Importance for stent design. Clin. Hemorheol. Microcirc. 2015, 59, 219-233.

(31) Chen, K.-Y.; Kuo, J.-F. Surface characterization and platelet adhesion studies of aliphatic polyurethanes grafted by fluorocarbon oligomers: effect of fluorocarbon chain length and carboxylic acid group. J. Mater. Sci.: Mater. Med. 2002, 13, 37-45.

(32) Fujisawa, N.; Odell, R. A.; Poole-Warren, L. A.; Bertram, C. D.; Woodard, J. C.; Schindhelm, K. Acute cellular interaction with textured surfaces in blood contact. J. Biomed. Mater. Res. 2000, 52, 517-527.

(33) Weisenberg, B. A.; Mooradian, D. L. Hemocompatibility of materials used in microelectromechanical systems: platelet adhesion and morphology in vitro. J. Biomed. Mater. Res. 2002, 60, 283-291.

(34) Clauser, J. C.; Maas, J.; Arens, J.; Schmitz-Rode, T.; Steinseifer, U.; Berkels, B. Automation of hemocompatibility analysis using image segmentation and supervised classification. Eng. Appl. Artif. Intell. 2021, 97, No. 104009.

(35) Rauwendaal, C. Polymer Extrusion, 1st ed.; Carl Hanser Fachbuchverlag, München, 2014.

(36) Nadzeyka, I.; Bolle, E.; Moos, M.; Kunitz, P.; Steinseifer, U.; Schmitz-Rode, T. Process analysis of spray atomization of dissolved polymers for manufacturing of blood-compatible textile implants. $J$. Ind. Text. 2017, 48, 926-940.

(37) Clauser, J.; Gester, K.; Roggenkamp, J.; Mager, I.; Maas, J.; Jansen, S. V.; Steinseifer, U. Micro-structuring of polycarbonateurethane surfaces in order to reduce platelet activation and adhesion. J. Biomater. Sci., Polym. Ed. 2014, 25, 504-518.

(38) Clauser, J. C. A New Analysis Method for Optical In-Vitro Hemocompatibility Assessment; Shaker, 2019.

(39) Hopmann, C.; Windeck, C.; Petzinka, F. In Microstructuring Polycarbonate Films by Variothermal Extrusion Embossing, AIP Conference Proceedings, AIP Publishing, Melville, 2016; 030016.

(40) Vollert, C. „Immature platelet fraction": Evaluation eines neuen Thrombozytenparameters in der Differentialdiagnose von Thrombozytopenien bei Kindern. Dissertation, 2013.

(41) Bundesaertzekammer Richtlinie der Bundesärztekammer zur Qualitätssicherung laboratoriumsmedizinischer Untersuchungen. In Deutsches Ärzteblatt; Deutscher Ärzteverlag GmbH, Berlin, 2014; pp $1583-1618$.

(42) Khorasani, M. T.; Mirzadeh, H. In vitro blood compatibility of modified PDMS surfaces as superhydrophobic and superhydrophilic materials. J. Appl. Polym. Sci. 2004, 91, 2042-2047.

(43) Chandy, T.; Das, G. S.; Wilson, R. F.; Rao, G. H. R. Use of plasma glow for surface-engineering biomolecules to enhance bloodcompatibility of Dacron and PTFE vascular prosthesis. Biomaterials 2000, 21, 699-712.

(44) Freeman, J.; Chen, A.; Weinberg, R. J.; Okada, T.; Chen, C.; Lin, P. H. Sustained Thromboresistant Bioactivity with Reduced Intimal Hyperplasia of Heparin-Bonded Polytetrafluoroethylene Propaten Graft in a Chronic Canine Femoral Artery Bypass Model. Ann. Vasc. Surg. 2018, 49, 295-303.

(45) Spiller, D.; Losi, P.; Briganti, E.; Sbrana, S.; Kull, S.; Martinelli, I.; Soldani, G. PDMS content affects in vitro hemocompatibility of synthetic vascular grafts. J. Mater. Sci.: Mater. Med. 2007, 18, 10971104.

(46) Sun, T.; Tan, H.; Han, D.; Fu, Q.; Jiang, L. No Platelet Can Adhere - Largely Improved Blood Compatibility on Nanostructured Superhydrophobic Surfaces. Small 2005, 1, 959-963. 